\title{
Kawasaki-Like Disease, a New Phenotype in Sars-CoV-2?
}

Beatriz Sordi Chara, ${ }^{\circledR}$ Júlia Machado Rickli, ${ }^{\circledR}$ Caroline Figueiredo da Silva, ${ }^{\circledR}$ Gabriela Pomaleski, ${ }^{\circledR}$ Rafael de March Ronsoni, ${ }^{\circledR}$ Marcus Vinícius Magno Gonçalves ${ }^{\circledR}$

Universidade da Região de Joinville, Departamento de Medicina, Joinville, SC - Brazil

\section{Abstract}

The coronavirus disease (COVID-19), that assumed pandemic proportions in March 2020, mainly affects the respiratory tract, causing severe interstitial pneumonia in adults. Worldwide data indicate that COVID-19 tends to be more benign in children, which is evidenced by a high incidence of asymptomatic or mild upper airways' infection cases in this population. However, recent studies have been associating Kawasaki-like symptoms as a nonclassical presentation of coronavirus disease in pediatrics. It is suggested that the intense cytokine cascade, promoted by the SARS-CoV-2 infection, can trigger a multisystem inflammatory response as an atypical Kawasaki form in genetically predisposed individuals. In this context, patients may develop more severe clinical features with a greater predisposition to myocardial involvement, Macrophage Activation Syndrome, and Kawasaki Disease Shock Syndrome. Despite critical conditions, patients usually respond to conventional treatment of Kawasaki Disease with intravenous immunoglobulin. This article intends to provide an approach to the association between Kawasaki-Like Syndrome and COVID-19.

\section{Background}

The Sars-CoV-2 virus was initially isolated in the city of Wuhan, China, during a pneumonia outbreak of unknown cause in December 2019, a disease then

\section{Keywords}

COVID-19; Betacoronavirus; Pandemics; Mucocutaneous Lymph Node Syndrome; Respiratory System/complications; Phenotype. named COVID-19. Due to its progressive worldwide spread, the World Health Organization decreed, three months later, the state of pandemic. ${ }^{1}$ Sars-CoV-2 usually affects the respiratory tract, causing severe interstitial pneumonia in adults. In children, however, it tends to be more benign, manifesting asymptomatically or as a mild infection of upper airways. A systematic review that assessed a 12-case series of children from China found that $39 \%-82 \%$ of the patients with COVID-19 developed a moderate course of the disease. ${ }^{2}$ A minority of pediatric cases presented severity, with respiratory failure, shock, coagulation dysfunction, and renal injury. ${ }^{3,4}$ Data from many countries indicate that the rate of Sars-CoV-2 infection in the population under 18 is low, ranging from $1 \%-2 \%$. Among the cases, the occurrence of unfavorable outcomes is even rarer. A cohort study by DeBiasi et al., ${ }^{5}$ with 177 young patients diagnosed with coronavirus disease found that $44(24.8 \%)$ patients needed hospitalization, among these, only $9(2.8 \%)$ were critically ill. ${ }^{5}$ Interestingly, one of them developed hypotension and myocardial depression associated with signs of hyperinflammatory state, a Kawasaki-like presentation. Despite being an unusual manifestation, the increase in the number of reported associations between Kawasaki Disease (KD) and COVID-19 in children raises new concerns about its consequences. ${ }^{5,6}$

Search Methods: In order to develop a nonsystematic narrative review, we executed literature searches in multiple databases (Google Scholar, PubMed, SciELO, and Medscape) with no time restriction for articles published in English. Search terms included the keywords: "Sars-CoV-2", “COVID-19", "Kawasaki-like”, "MIS-C", "PIMS-TS" and "Autoimmune disease". We emphasized in case reports, clinical trials, systematic and nonsystematic reviews, and guidelines aiming to

Mailing Address: Beatriz Chara

Rua Paulo Malschitzki, s/n, Zona Industrial Norte. Postal Code: 89219-710, Joinville, SC - Brazil.

E-mail: beatrizsordichara@gmail.com 
provide an overview of the main aspects of Kawasaki-like syndrome following Sars-CoV-2 infection.

\section{Discussion}

\section{Definition}

$\mathrm{KD}$ is a rare systemic medium-vessel acute vasculitis of the childhood of undefined etiology. It usually has a predilection for coronary arteries and its main complication is the formation of aneurysms. The disease typically occurs in children between 5 months and 5 years of age and is more incident in Asian descendants. According to the American Heart Association (AHA), its diagnosis involves persistent fever associated with five other criteria: oral mucosal changes, bilateral nonexudative conjunctivitis, exanthematous rash, flaking of hands and feet, and cervical lymphadenopathy. ${ }^{7}$ Among its laboratory findings, anemia, leukocytosis, and thrombocytosis can be detected from the 7th day of fever, in addition to hypoalbuminemia and increased transaminases. Other complementary exams, such as echocardiography, can demonstrate coronary aneurysms or cardiac dysfunction (left ventricular function depression, mitral valve regurgitation or pericardial effusion). ${ }^{3} \mathrm{New}$ studies suggest a potential correlation between infectious agents, like Sars-CoV-2, and the inflammatory cascade that results in KD. ${ }^{3}$ The first report of KD in a patient with COVID-19 was a 6-month-old female child who manifested fever, tachycardia, signs of respiratory distress, persistent polymorphic maculopapular rash and sparing conjunctivitis, with no echocardiogram alterations. She was treated with intravenous immunoglobulin (IVIG) and high-dose aspirin, achieving clinical resolution. ${ }^{4}$ Since then, numerous cases associating both diseases have been related. A publication by the Royal College of Paediatrics (2020) proposed that this presentation be called "Pediatric Inflammatory Multisystem Syndrome Temporally Associated with Sars-CoV-2" (PIMS-TS). ${ }^{8}$ The US Center for Disease Control ${ }^{9}$ has assigned the name "Multisystem Inflammatory Syndrome in Children" (MIS-C) for the same condition. ${ }^{9}$ According to them, clinical aspects of PIMS-TS/MIS-C may be similar to an atypical $\mathrm{KD}$, including prolonged fever, skin rash, high levels of inflammatory biomarkers, lymphadenopathy, gastrointestinal symptoms, and multiple organ dysfunction. ${ }^{8,9}$

\section{Epidemiology}

A retrospective cohort study conducted by Verdoni et al. ${ }^{3}$ in Bergamo, Italy, between 2015 and April 2020, found a 30 times higher monthly incidence of KD symptoms during the coronavirus disease outbreak when compared to previous years. In terms of epidemiology, Asian children are more likely to have the typical form of KD. However, the Kawasaki-like syndrome associated with SARS-COV-2 infection seems to affect Afro-Caribbean descent more often than expected. In a cohort study by Riphagen et al. ${ }^{10}$, carried out in a pediatric population diagnosed with coronavirus disease in the United Kingdom, six children (75\%) had Afro-Caribbean origin, which may suggest a genetic susceptibility or a higher rate of COVID-19 in this offspring. ${ }^{10}$

\section{Pathogenesis}

Concerning tissue damage in COVID-19, PIMS-TS/ MIS-C appears to be mostly mediated by the host innate immunity. The "cytokine storm" provided by Sars-CoV-2 infection, that is similar to macrophage activation in viral-induced hemophagocytic lymphohistiocytosis and resembles Macrophage Activation Syndrome (MAS) in KD, might be a trigger to Kawasaki-like response, regarding the hypothesis of KD deriving from an aberrant immune response to a pathogen in genetically predisposed individuals. ${ }^{3}$ It is suggested that its mechanism is based on a post-inflammatory antibody-mediated reaction, therefore, the identification of viral replication has not proved to be truly relevant in these cases. ${ }^{6}$

\section{Clinical Aspects}

Compared to classic KD, PIMS-TS/MIS-C presents a later onset, with an average of 9 versus 3 years of age. Moreover, fever and asthenia are prevalent related features in this context. ${ }^{9,11}$ Kawasaki-like syndrome associated with Sars-CoV-2 also comprises increased incidence of gastrointestinal manifestations, including abdominal pain, mesenteric lymphadenopathy, vomiting, diarrhea or even acute abdomen, demanding surgery. ${ }^{9,12}$ In the study of Bergamo, by Verdoni et al., ${ }^{3}$ patients who had Kawasaki disease presentations during the COVID-19 pandemic had more leucopenia with marked lymphopenia, thrombocytopenia, increased ferritin, and abnormalities in the echocardiogram, than KD cases from previous years. $^{3}$ 
Along with a higher occurrence of MAS and Kawasaki Disease Shock Syndrome (KDSS), myocardial injury has also been more frequent in PIMS-TS/MIS-C. ${ }^{9,13}$ Another divergence from typical KD is that patients with MIS-C often have low blood pressure due to reduced left ventricular systolic function. ${ }^{9}$ An analysis of 35 children with MIS-C by Belhadjer and colleagues (2020), detected an important reduction in ventricular ejection in $1 / 3$, and the presence of coronary artery dilation in $17 \%$. In addition, $80 \%$ of these patients needed inotropic support. In the same study, the presence of the "cytokine storm" was evidenced in all children by the high levels of C-reactive protein, Interleukin-6, and D-dimer, indicating macrophage activation. ${ }^{9}$ Although aneurysms have been less observed than in previous cases of $\mathrm{KD}$, it is worth mentioning that this is a possible late complication and must be investigated in followup evaluations. ${ }^{9}$

\section{Diagnosis}

Sars-CoV-2 infection can be detected by nasopharyngeal and oropharyngeal PCR swab in the first 5-7 days of disease, and from the 10th to 15th day of symptoms, qualitative antibody tests (IgM and $\operatorname{IgG}$ ) can be performed. ${ }^{3}$ According to the pathophysiology of PIMSTS/MIS-C, based on an exacerbated inflammatory reaction mediated by immunoglobulins, diagnosis is best established by serology, rather than RT-PCR, which was also demonstrated by 8 out of 10 patients with Kawasaki-like Syndrome studied by Verdoni et al. (2020), who had positive antibodies against SarsCoV-2, IgG, IgM or both. ${ }^{3}$ It is important to note that not all antibody tests for coronavirus are highly accurate. False negative results are frequently obtained in low sensitivity tests, and false positives for Sars-CoV-2 IgG can occur due to a cross reactivity of other preexisting immunoglobulins, especially in patients with disorders of hyperinflammatory state, such as KD. ${ }^{12}$

\section{Treatment}

In order to control the exacerbated immune response, and based on the standard treatment for KD, IVIG has been used as the main therapy for PIMS-TS/MIS-C. Its exact mechanism is still not well understood, but it presumably works by reducing the action of monocytes, macrophages and other inflammatory cells, preventing the progression of myocardial damage. Positive outcomes have been established in several studies. Belhadjer et al. ${ }^{9}$, for example, detected restored ventricular function in 25 of the 35 MIS-C patients treated with immunoglobulins. ${ }^{9}$ Other supportive treatments include antibiotics, anticoagulants, corticosteroids, like methylprednisolone, and anti-inflammatories, such as aspirin, which can be used concomitantly with IVIG to reduce the risk of immunoglobulin resistance, especially in MAS and KDSS. ${ }^{3,9,13}$ For cases unresponsive to IVIG, anti-interleukin 1 and anti-interleukin 6 immunomodulators, like Anakinra and Tocilizumab, are therapeutic options. ${ }^{14}$ Most of the children achieve clinical improvement quickly, on average in 4 days, but more critical cases may require longer hospitalization with mechanical ventilation, administration of vasopressors, or extracorporeal circulation. ${ }^{10,13}$

\section{Conclusion}

The clinical spectrum of COVID-19 in pediatrics is not clearly defined. However, new studies indicate that children may experience an acute cardiac decompensation related to the inflammatory response that follows Sars$\mathrm{CoV}-2$ infection. Its potential Kawasaki-like presentation evidences the heterogeneity of clinical manifestations and the need to test patients with non-classic symptoms. ${ }^{4}$ Reports of complete recovery of cardiac function due to immunoglobulin therapy support the importance of early diagnosis and treatment., ${ }^{7,9}$ The adjunctive use of steroids and anti-inflammatories has also been shown relevant in more critical features, such as MAS and KDSS, ensuring a better prognosis. Although multisystem inflammation syndrome is still rare, affecting about $0.1 \%$ of children exposed to Sars-CoV-2, it is important for the medical community to be aware of this new phenotype and its immunological mechanisms to anticipate the identification of the most severe cases..$^{3,6}$

\section{Author contributions}

Conception and design of the research: Ronsoni RM, Magno Gonçalves MV. Acquisition of data: Chara BS, Rickli JM, da Silva CF, Pomaleski G. Analysis and interpretation of the data: Chara BS, Rickli JM, da Silva CF, Pomaleski G. Statistical analysis: Chara BS, Rickli JM, da Silva CF, Pomaleski G. Writing of the manuscript: Chara BS, Rickli JM, da Silva CF, Pomaleski G. Critical revision of the manuscript for intellectual content: Ronsoni RM, Magno Gonçalves MV. 


\section{Potential Conflict of Interest}

No potential conflict of interest relevant to this article was reported.

\section{Sources of Funding}

There were no external funding sources for this study.

\section{References}

1. World Health Organization (WHO) announces COVID-19 outbreak a pandemic. [Cited in 2029 Dec 13] Euro.who.int. 2020. Available from: http://www.euro.who.int/en/health-topics/health-emergencies/ coronavirus-covid-19/news/news/2020/3/whoannounces-covid-19inflam-a-pandemic

2. Streng A, Hartmann K, Armann J, Berner R, Liese J. COVID-19 bei hospitalisierten Kindern und Jugendlichen. Monatsschrift Kinderheilkunde. 2020;1-12. doi:10.1007/s00112-020-00919-7

3. Verdoni L, Mazza A, Gervasoni A, Martelli L, Ruggeri M, Ciuffreda M et al. An outbreak of severe Kawasaki-like disease at the Italian epicentre of the SARS-CoV-2 epidemic: an observational cohort study. Lancet. 2020:395(0239):1771-8. doi:10.1016/S0140-6736(20)31103-X

4. Jones VG, Mills M, Suarez D, Hogan CA, Yeh D, Segal JB, et al. COVID-19 and Kawasaki Disease: Novel Virus and Novel Case. Hosp Pediatr. 2020.;10(6):537-40. doi:10.1542/hpeds.2020-0123

5. DeBiasi R, Song X, Delaney M, Bell M, Smith K, Pershad J et al. Severe COVID-19 in Children and Young Adults in the Washington DC Metropolitan Region. J Pediatr.2020;223:199-203e1 doi: 10.1016/j. jpeds.2020.05.007

6. Viner RM, Whittaker E. Kawasaki-like disease: emerging complication during the COVID-19 pandemic. Lancet. 2020.;395(10239):1741-3. doi:10.1016/S0140-6736(20)31129-6

7. Sandhaus H, Crosby D, Sharma A, Gregory SR. Association Between COVID-19 and Kawasaki Disease: Vigilance Required from Otolaryngologists. Otolaryngology Head Neck Surg. 2020. 163(2):316-7. doi:10.1177/0194599820930238

\section{Study Association}

This study is not associated with any thesis or dissertation work.
8. Royal College of Paediatrics and Child Health. Guidance-Paediatric multisystem inflammatory syndrome temporally associated with COVID-19. RCPCH; 2020. [Cited in 202012 Dec] Available from: https://www.rcpch.ac.uk/resources/guidance-paediatricmultisysteminflammatory-syndrome-temporallyassociated-covid-19

9. Belhadjer Z, Méot M, Bajolle F, Khraiche D, Legendre A, Abakka S, et al Acute heart failure in multisystem inflammatory syndrome in children (MIS-C) in the context of global SARS-CoV-2 pandemic.Circulation. 2020;142(5):429-36. doi: 10.1161/CIRCULATIONAHA.120.048360

10. Riphagen S, Gomez X, Gonzalez-Martinez C, Wilkinson N, Theocharis P. Hyperinflammatory shock in children during COVID-19 pandemic. Lancet. 2020395(10237):1607-8. doi:10.1016/ S0140-6736(20)31094-1

11. Whittaker E, Bamford A, Kenny J, Kaforou M, Jones CE, Shah P, et al. Clinical Characteristics of 58 Children with a Pediatric Inflammatory Multisystem Syndrome Temporally Associated With SARS-CoV-2 JAMA. 2020;324(3):259-69. doi:10.1001/jama.2020.10369

12. Toubiana J, Poirault C, Corsia A, Bajolle F, Fourgeaud J, Angoulvant F. Kawasaki-like multisystem inflammatory syndrome in children during the covid-19 pandemic in Paris, France: prospective observational study. BMJ. 2020;369:m2094. Published 2020 Jun 3. doi:10.1136/bmj.m2094

13. European Centre for Disease Prevention and Control. Paediatric inflammatory multisystem syndrome and SARS-CoV-2 infection in children. ECDC: Stockholm; 2020. [Cited in 202016 Nov] 